\section{International Scientific Journal Theoretical \& Applied Science}

\author{
p-ISSN: 2308-4944 (print) e-ISSN: 2409-0085 (online) \\ $\begin{array}{lll}\text { Year: } 2016 \quad \text { Issue: } 7 & \text { Volume: } 39\end{array}$ \\ Published: $30.07 .2016 \quad$ http://T-Science.org
}

Sergey Alexandrovich Mishchik Associate Professor, Candidate of Pedagogical Science, Corresponding member of International Academy TAS, Assistant professor Department of Physics, State Maritime University Admiral Ushakov, Russia, sergei_mishik@mail.ru

SECTION 21. Pedagogy. Psychology. Innovation in Education

\title{
ANALYSIS OF BASIC PEDAGOGOMETRIC MATHEMATICAL MODELS OF EDUCATIONAL ACTIVITY
}

\begin{abstract}
: the main directions of analysis of basic pedagogometric mathematical models of educational activity recorded in the form of differential equations to the process of identity formation, set highlighted training simulation activity classes - static, dynamic-cal, based on fuzzy logic and neural networks set the basic heuristic potential of education-enforcement process in terms of information and communication online media, as well as the use of the twelve pointed star Ertsgammy as a leading formative processes relative-enforcement hyperspace of life, as well as psychological and pedagogical activity theory, psycho-pedagogical system analysis and the theory of the formation of mental actions.

Key words: basicity, pedagogometric mathematical modeling, pedagogometric, consistency, integrity, the subject of activity, personality analysis, star Ertsgammy, educational space.

Language: Russian

Citation: Mishchik SA (2016) ANALYSIS OF BASIC PEDAGOGOMETRIC MATHEMATICAL MODELS OF EDUCATIONAL ACTIVITY. ISJ Theoretical \& Applied Science, 07 (39): 107-111.

Soi: http://s-o-i.org/1.1/TAS-07-39-19 Doi: crossef http://dx.doi.org/10.15863/TAS.2016.07.39.19
\end{abstract}

\section{УДК 372.851}

\section{АНАЛИЗ БАЗИСНЫХ ПЕДАГОГОМЕТРИЧЕСКИХ МАТЕМАТИЧЕСКИХ МОДЕЛЕЙ УЧЕБНОЙ ДЕЯТЕЛЬНОСТИ}

Аннотация: Рассмотрены основные направления анализа базисных педагогометрических математических моделей учебной деятельности записанных в виде систем дифференциальных уравнений к прочессу формирования личности, устанавливаются выделяются классы моделирования учебной деятельности - статические, динамические, на основе нечеткой логики и нейронных сетей к анализу образовательного процесса в условиях информационно-коммуникативной интернет-среды, а также применение двенадиати конечной звезды Эригаммы в качестве ведущего формообразовательного процесса относительно гиперпространства жизнедеятельности, а также психолого-педагогической теории деятельности, психолого-педагогического системного анализа и теории формирования умственных действий.

Ключевые слова: базисность, педагогометрическое математическое моделирование, педагогометрика, системность, ичелостность, субъект деятельность, личность, анализ, звезда Эригаммы, образовательное пространство.

Анализ базисных педагогометрических математических моделей учебной деятельности в современной практике рассматривают как проблему разработки математических моделей и программного комплекса для задач управления непрерывными образовательнотехнологическими процессами. Дальнейшая информатизация учебной деятельности студентов требует проектирования и применения автоматизированных систем управления на базе компьютерных сетей и информационных систем, что способствует созданию технологий сбора и обработки информации о результатах учебной деятельности в электронном виде.

Существющее математическое моделирование непрерывной учебной деятельности связывается с общим методом анализа моделей, записанных в виде систем 
дифференциальных уравнений, или выполнением отдельных видов учебных действий. Разработка систем дифференциальных уравнений являются более адекватными математическими моделями целостного моделирования учебной деятельности. Моделирование отдельных учебных действий студентов в начальной фазе учебного знания приводит к задачам линейного или динамического программирования. Непрерывность процесса преобразования учебных действий в навыки и учебную деятельность является базисным условием математического моделирования учебной деятельности.

В целом, выделяют четыре класса моделирования учебной деятельности статические, динамические, на основе нечеткой логики и нейронных сетей. При этом выделяют критерии задач оптимизации и считают, что наиболее разработанным подклассом статических и динамических моделей являются линейные модели; динамические модели представляются более адекватными, чем статические; стохастические модели являются более адекватными, чем детерминированные, а актуальность моделей на основе нечеткой логики и нейронных сетей увеличивается [16] .

Предпринимаются попытки предложить особенную методику формирования учебного плана. Предлагаемые алгоритмы основаны на применении математического аппарата балансовой модели для повышения качества планирования учебного процесса при учёте установленных между тематических связей в изучаемых дисциплинах. Развиваемая возможность усовершенствования процесса составления учебных планов на основе применения методов математического моделирования формируется на основе вычислительных алгоритмов, их теоретическом обосновании и практической реализации в виде программного комплекса для автоматизированного формирования рабочего учебного плана [ 2 ] .

Атрибутивность математических моделей учебной деятельности проявляется у студентов в процессе изучения прикладной математики, направленной на решение учебнопрофессиональных задач, проектирующих собственную учебную деятельность, при которой методические основы моделирования с помощью графов в процессе обучения являются собственным условием формирования самой учебной деятельности посредством объединения отдельных моделей в методическую систему моделирования с помощью графов; обоснованы и разработаны средства реализации методической системы моделирования с помощью графов. определены и характеризованы основные этапы построения графовых моделей учебной деятельности [ 1 ] .

Дальнейшее развитие математического моделирования учебной деятельности связывается с развитием дистанционных форм образования. Поэтому в разработке математической и информационной моделей обучающего курса применяют объектноориентированный и системный подходы; методики эффективного создания многоуровневого обучающего курса. Математическое моделирование процесса обучения требует: реализации сквозного подхода к проектированию и разработке обучающей системы на основе общесистемных принципов; моделировании процесса обучения с использованием сетей Петри и цепей Маркова для расчета вероятностных характеристик процесса обучения.

При этом представляется, что объектноориентированный подход к построению модели обучающего курса позволяет эффективно хранить и использовать такие объекты, как: курс, частично представляемый сетью Петри; трек обучения (траектория обучаемого по сети Петри); тезаурус обучаемого и модель текущих знаний; профиль курса, строящийся на основе данных, хранящихся в треках, и позволяющий анализировать спроектированный курс с применением теории цепей Маркова и корректировать его с целью повышения эффективности процесса обучения [3].

Оценить уровень совершенствования математических моделей учебной деятельности предлагается проводить с использованием непараметрических методов математической статистики: критериев хи-квадрат и знаков. Этот процесс будет успешным при использовании параметров (показателей), удовлетворяющих общим функциям образовательного процесса. К ним относят коэффициенты общего интеллекта, показатели тревожности и эмоциональности, а также отметки по итоговым контрольным работам [ 15 ].

Относительно анализа базисных педагогометрических математических моделей учебной деятельности проводится исследование по применению в образовательном процессе: вероятностных методов по выделению конкретных свойств и связей отдельных элементов образовательного процесса, когда неоднозначность педагогических процессов имеет вероятностный характер; графовых методов по определению элементов образовательного процесса, которые обладают структурой моделируемого образовательного процесса; алгебраических методов по установлению элементов образовательного процесса, в котором необходима формализация 
больших объемов информации; методов линейного программирования по вычислению количественной оценки результатов образовательной деятельности; методов теории игр по представлению элементов образовательного процесса, в котором каждое образовательное действие может дать в разных случаях разные результаты с известной вероятностью.

При этом обобщенный алгоритм применения отдельных методов математического моделирования в обучении представляет этапы: построения модели элементов образовательного процесса; формирующий учебный эксперимент; объяснение результатов на языке дидактики и установление соответствия вывода о свойствах модели образовательных объектов [4] .

Анализ базисных педагогометрических математических моделей учебной деятельности относительно психолого-педагогического системного анализа, психологической теории деятельности, теории формирования интеллекта, гиперпространства целостно-системных циклов жизнедеятельности эрцгамного формообразования составляет особую задачу педагогометрики. Целостно-системное учебное действие (ЦСУД) составляет базисную структурную основу целостно-системного цикла жизнедеятельности (ЦСЦЖ), состоящего из двенадцати компонентов звезды Эрцгаммы. Каждый элемент ЦСЦЖ можно представить методами системного анализа через двенадцать психолого-педагогических действий, которые в процессе интериоризации принимают двенадцать основных форм от ориентационной до внутренней и также имеют деятельностную основу. С учётом процессов коммуникативной деятельности дополнительно выделяются четыре целостно-системные учеб-ные действия. Существует сорок базисных ЦСУД, которые имеют предметно-деятельностную основу относительно ЦСЦЖ, психолого-педагогического системного анализа и процесса формирования интеллекта. Математическое моделирование целостно-системного учебного действия определяет базисную задачу педагогометрики $[5,6]$.

Любое целостно-системное учебное действие имеет три базисные компонента: ориентировочный, исполнительный и контрольный, которые определяют основные направления математического моделирования ЦСУД. Множество элементов учебного действия можно записать в виде набора последовательных элементов системных операций. Каждый элемент ЦСУД характеризуется конкретными свойствами, которые однозначно определяют его в данной системе. Совокупность всех свойств элемента учебного действия устанавливает его состояние. Между базисными компонентами ЦСУД существует связь - множество зависимостей свойств одного элемента от свойств других элементов системы учебного действия. Множество связей между элементами учебного действия можно представить в виде треугольных, базисных свяей. Зависимость свойств элементов учебного действия имеет двусторонний взаимосвязанный характер. Это определяет структуру системы учебного действия множество элементов системы и связей между ними $[7,8]$.

Структура ЦСУД зависит от статического и динамического состояний. В условиях статического поведения учебного действия связь между ориентировочным и контрольным компонентами представляется как связь между функциями входа и выхода системы без учета предыдущих ее состояний. В условиях динамического состояния учебного действия система зависит не только от функций входов, но и от функций переходов. В данном случае можно определить обобщенный показатель качества целостно-системного учебного действия как вектор, компоненты которого есть частные показатели отдельных свойств ЦСУД. Размерность $\mathrm{n}$ определяется числом системных свойств учебного действия $[9,10,11]$.

При определении обобщенного показателя качества целостно-системного учебного действия надо применять не натуральные частные показатели, а нормированные значения. Это обеспечивает приведение показателей к одному масштабу - через «абсолютное» значение каждого показателя ЦСУД. Любое целостносистемное учебное действие можно принять за идеальную систему, если её гипотетическая модель, удовлетворяет всем критериям системного качества. Тогда выделяется область адекватности ЦСУД - окрестность значений показателей целостно-системных свойств учебного действия. Радиус адекватности имеет нормированное значение, которое определяется целостной зависимостью.

Все критерии качества целостно-системного учебного действия определяются тремя типами: критерий пригодности (радиус области адекватности соответствует допустимым значениям всех частных показателей); критерий оптимальности (существует хотя бы один частный показатель качества, значения которого принад-лежат области адекватности с оптимальным радиусом ) и критерий превосходства (если значения частных показателей качества принадлежат области адекватности с оптимальным радиусом по всем показателям). Все критерии качества ЦСУД 
обладают свойствами представительности, эластичности и простоты $[12,13,14]$.

При прогнозировании процесса развития целостно-системного учебного действия применяется фактографический метод - метод наименьших квадратов (МНК). При этом анализируется процесс наращивания базисных компонентов ЦСУД относительно целостности и системности - ориентировки, исполнения и контроля через систему временного ряда. Поэтому в дальнейшем ЦСУД будет развиваться в соответствии с законом развития педагогометрических характеристик, связанных с расчетными значения исходного ряда, его фактическими значениями и числом наблюдений. $\mathrm{C}$ учётом адаптации к новым условиям необходимо ввести коррективы в прогнозные оценки развития ЦСУД через коэффициенты дисконтирования, которые характеризует изменение ценности информации во времени. При прогнозной оценке устанавливается и дальность прогнозирования с учётом абсолютного времени упреждения и величиной эволюционного цикла развития ЦСУД. Предложенное математическое моделирование целостно-системного учебного действия устанавливает одну из базисных задач педагогометрики, представляющей процесс математического моделирования подготовки широкопрофильных специалистов и их личностных качеств.

\section{References:}

1. Debol'skii TA (2004) Methodical bases fashionlation with the help of graphs in the process of math-trained cadets of military-technical college [Text]: dis. ... Cand. ped. Sciences:13.00.02 / T. A. Debol'skii. Yaroslavl, 2004. - $192 \mathrm{p}$.

2. Demakov VI (2006) Mathematical modeling of the process of creation of curricula for higher education [Text]: dis. ... Cand. those. Sciences: 05.13.18 / V. I.Demakov. - Irkutsk, 2006. - 127 p.

3. Dorofeev AS (2006) Model training course and implementation of the program cover a distance-tional learning: systems and sub-object moves [Text]: Auto-Ref. Dis. ... Cand. those. Sciences: 05.13.01 / AS Dorofeev. - Irkutsk, 2006. - 15 p.

4. Kiseleva OM (2007) Application of methods of mathematical-modeling techniques in teaching: Auto-Ref. Dis. ... Candidate. ped. Sciences: Smolensk, 2007. - 19 p.

5. Mishchik SA (2014) Pedagogometrika and mathematical modeling educational activity. Materialy Mezhdunarodnoy nauchnoy konferenctsii "Modern mathematics in science" - 30.06.2014. ISJ Theoretical \&Applied Science 6(14): 54-56 Caracas, Venezuela. doi:

http://dx.doi.org/10.15863/TAS.2014.06.14.10

6. Mishchik SA (2014) Simulation training activity methods of mathematical logic. Materialy Mezhdunarodnoy nauchnoy konferenctsii "Eurapean Science and Education" - 30.07.2014. ISJ Theoretical \&Applied Science 6(15): 72-74 Marseille,
France.

doi: http://dx.doi.org/10.15863/TAS.2014.07.15.13

7. Mishchik SA (2014) Mathematical modeling system integrity-cycle of life activity - first goal pedagogometriki. Materialy Mezhdunarodnoy nauchnoy konferenctsii "European Applied Sciences" - 30.08.2014. ISJ Theoretical \&Applied Science 7(16): 77-79. Aix-en-Provence, France. doi: http://dx.doi.org/10.15863/TAS.2014.08.16.13

8. Mishchik SA (2014) Mathematical modeling system integrity-curricular activities - the second problem pedagogometriki. Materialy Mezhdunarodnoy nauchnoy konferenctsii "European Innovation" - 30.09.2014. ISJ Theoretical \&Applied Science 9(17): 126-128 Martigues, France. doi: http://dx.doi.org/10.15863/TAS.2014.09.17.21

9. Mishchik SA (2014) Mathematical modeling holistic-systemic communicative activity - the third task pedagogometriki. Materialy Mezhdunarodnoy nauchnoy konferenctsii "European Scientific Achievements" 30.10.2014. ISJ Theoretical \&Applied Science 10(18): 45-47 Brighton, UK. doi: http://dx.doi.org/10.15863/TAS.2014.10.18.11

10. Mishchik SA (2014) Mathematical modeling integrity - system performance subject - fourth task pedagogometriki. Materialy Mezhdunarodnoy nauchnoy konferenctsii "Eurapean Science and Technology" 30.11.2014. ISJ Theoretical \&Applied Science 11(19): 51-54 Southampton, UK. doi: http://dx.doi.org/10.15863/TAS.2014.11.19.10 


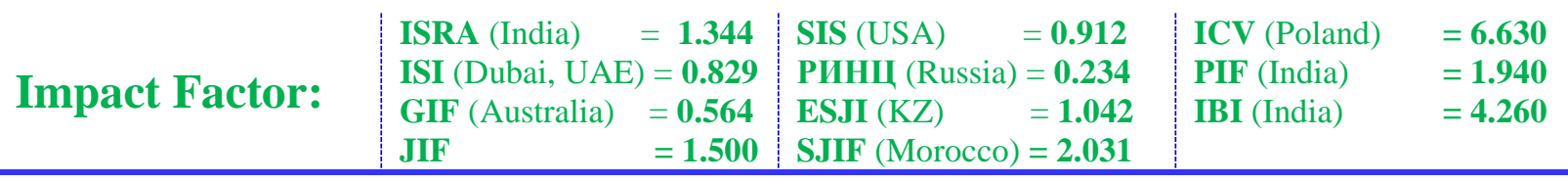

11. Mishchik SA (2015) Pedagogometrik - science and academic subject. Materialy Mezhdunarodnoy nauchnoy konferenctsii "European Technology in Science" 28.02.2015. ISJ Theoretical \& Applied Science 02 (22): 103-106 Malmö, Sweden. doi: http://dx.doi.org/10.15863/TAS.2015.02.22.17

12. 12.Tokmazov GV (2014) Matematicheskoe modelirovanie $\mathrm{v}$ uchebno-professional'noy deyatel'nosti. Materialy Mezhdunarodnoy nauchnoy konferentsii «Modern mathematics in science» - 30.06.2014. ISJ Theoretical \& Applied Science 6(14): 44-46. - Caracas, Venezuela. doi: http://dx.doi.org/10.15863/TAS.2014.06.14.8

13. Tokmazov GV (2014) Analysis says study skills in the study of mathematics, Materialy Mezhdunarodnoy nauchnoy konferenctsii "Eurapean Science and Education" 30.07.2014. ISJ Theoretical \&Applied Science 7(15): 72-74 Marseille, France. doi: http://dx.doi.org/10.15863/TAS.2014.07.15.1
14. Tokmazov GV (2014) Mathematical modeling research skills in educational activity methods of probability theory. Materialy Mezhdunarodnoy nauchnoy konferenctsii "Eurapean Science and Technology" 30.11.2014. ISJ Theoretical \&Applied Science 11(20): 66-69 Southampton, United Kingdom. doi:

http://dx.doi.org/10.15863/TAS.2014.11.19.13

15. Chepelev PN (2003) Evaluation of teacherscal innovations using neparametical methods of mathematical statistics [Text]: dis. ... Cand. ped. Sciences: 13.00.01 / PN Chepelev. - Bratsk, 2003. - $199 \mathrm{p}$.

16. Shabayev AI (2005) Development of mathematical models of mo-and software for continuous process control problems [Text]: dis. ... Cand. those. Sciences: 05.13.18 / AI Shabayev. - Petrozavodsk, 2005. -177 p. 